\title{
MATROID PATHWIDTH AND CODE TRELLIS COMPLEXITY*
}

\author{
NAVIN KASHYAP ${ }^{\dagger}$
}

\begin{abstract}
We relate the notion of matroid pathwidth to the minimum trellis state-complexity (which we term trellis-width) of a linear code, and to the pathwidth of a graph. By reducing from the problem of computing the pathwidth of a graph, we show that the problem of determining the pathwidth of a representable matroid is NP-hard. Consequently, the problem of computing the trellis-width of a linear code is also NP-hard. For a finite field $\mathbb{F}$, we also consider the class of F-representable matroids of pathwidth at most $w$, and correspondingly, the family of linear codes over $\mathbb{F}$ with trellis-width at most $w$. These are easily seen to be minor-closed. Since these matroids (and codes) have branchwidth at most $w$, a result of Geelen and Whittle shows that such matroids (and the corresponding codes) are characterized by finitely many excluded minors. We provide the complete list of excluded minors for $w=1$, and give a partial list for $w=2$.
\end{abstract}

Key words. Matroids, pathwidth, linear codes, trellis complexity, NP-hard.

AMS subject classifications. 05B35, 94B05

1. Introduction. The notion of pathwidth of a matroid has received some recent attention in the matroid theory literature [7, 9. This notion has long been studied in the coding theory literature, where it is used as a measure of trellis complexity of a linear code [14, [5], 17. However, there appears to be no standard coding-theoretic nomenclature for this notion. It has been called the state complexity of a code in [10, but the use of this term there conflicts slightly with its use in [17. So to avoid ambiguity, we will give it a new name here - trellis-width — which acknowledges its roots in trellis complexity.

The relationship between matroid pathwidth and code trellis-width can be made precise as follows. To an arbitrary linear code $\mathcal{C}$ over a finite field $\mathbb{F}$, we associate a matroid, $M(\mathcal{C})$, which is simply the vector matroid, over $\mathbb{F}$, of any generator matrix of the code. Recall that in coding theory, a matrix $G$ is called a generator matrix of a code $\mathcal{C}$, if $\mathcal{C}$ is the rowspace of $G$. Consequently, the matroid $M(\mathcal{C})$ does not depend on the actual choice of the generator matrix, and so is a characteristic of the code $\mathcal{C}$. The code $\mathcal{C}$ may in fact be viewed as a representation over $\mathbb{F}$ of the matroid $M(\mathcal{C})$. The trellis-width of $\mathcal{C}$ is simply the pathwidth of $M(\mathcal{C})$; we will give the precise definition of matroid pathwidth in Section 2.2

It has repeatedly been conjectured in the coding theory literature that computing the trellis-width of a linear code over a fixed finite field $\mathbb{F}$ is NP-hard [10, [11, 17, Section 5]. This would imply that the corresponding decision problem (over a fixed finite field $\mathbb{F}$ ) - given a generator matrix for a code $\mathcal{C}$ over $\mathbb{F}$, and a positive integer $w$, deciding whether or not the trellis-width of $\mathcal{C}$ is at most $w$ - is NP-complete. This decision problem has been given various names - "Maximum Partition Rank Permutation" 10, "Maximum Width" 11] and "Trellis State-Complexity" [17.

An equivalent statement of the trellis-width conjecture above is the following: given a matrix $A$ over $\mathbb{F}$, the problem of computing the pathwidth the vector matroid $M[A]$ is NP-hard. In this paper, we prove the above statement for any fixed field $\mathbb{F}$,

${ }^{*}$ This work was supported in part by a research grant from the Natural Sciences and Engineering Research Council (NSERC) of Canada.

${ }^{\dagger}$ Dept. of Mathematics and Statistics, Queen's University, Kingston, ON, K7L 3N6, Canada. Email: nkashyap@mast.queensu.ca 
not necessarily finite. Our proof is by reduction from the problem of computing the pathwidth of a graph, which is known to be NP-hard 1, 2. Thus, in particular, computing the trellis-width of a linear code over $\mathbb{F}$ is NP-hard, which settles the aforementioned coding-theoretic conjecture.

The situation is rather different if we weaken the trellis-width decision problem above by not considering the integer $w$ to be a part of the input to the problem. In other words, for a fixed finite field $\mathbb{F}$, and a fixed integer $w>0$, consider the following problem:

given a length- $n$ linear code $\mathcal{C}$ over $\mathbb{F}$, decide whether or not $\mathcal{C}$ has trellis-width at most $w$.

The equivalent decision problem for matroid pathwidth would be to decide (for a fixed finite field $\mathbb{F}$ and integer $w>0$ ) whether or not a given $\mathbb{F}$-representable matroid has pathwidth at most $w$. Based on results from the structure theory of matroids [6], we strongly believe that these problems are solvable in polynomial time.

In the process of studying matroids of bounded pathwidth, we observe that for any finite field $\mathbb{F}_{q}=G F(q)$ and integer $w>0$, the class, $\mathcal{P}_{w, q}$, of $\mathbb{F}_{q}$-representable matroids having pathwidth at most $w$, is minor-closed and has finitely many excluded minors. As a relatively easy exercise, we show that the list of excluded minors for $\mathcal{P}_{1, q}$ consists of $U_{2,4}, M\left(K_{4}\right), M\left(K_{2,3}\right)$ and $M^{*}\left(K_{2,3}\right)$. Unfortunately, the problem of finding excluded-minor characterizations of $\mathcal{P}_{w, q}$ for $w>1$ becomes difficult very quickly. We give a list of excluded minors for $\mathcal{P}_{2, q}$, which is probably not complete.

The rest of the paper is organized as follows. In Section 2, we lay down the definitions and notation used in the paper. In Section 3, we prove that, for any fixed field $\mathbb{F}$, the problem of computing the pathwidth of an $\mathbb{F}$-representable matroid is NP-hard, and therefore, so is the problem of computing the trellis-width of a linear code over $\mathbb{F}$. Finally, in Section 4 we consider the class of matroids $\mathcal{P}_{w, q}$. We give the complete lists of excluded minors for $\mathcal{P}_{1, q}$ and the corresponding family of linear codes over $\mathbb{F}_{q}$ having trellis-width at most one. We also give a partial list of excluded minors for $\mathcal{P}_{2, q}$.

2. Preliminaries. We assume familiarity with the basic definitions and notation of matroid theory, as expounded by Oxley [15]. The main results and proofs in this paper will be given in the language of matroid theory, rather than that of coding theory, as it is easier to do so. However, as our results may be of some interest to coding theorists, we make an effort in this section to provide the vocabulary necessary to translate the language of matroid theory into that of coding theory. Definitions of coding-theoretic terms not explicitly defined here can be found in any text on coding theory (e.g., [13]).

2.1. Codes and their Associated Matroids. Let $\mathcal{C}$ be a linear code of length $n$ over the finite field $\mathbb{F}_{q}=G F(q)$. The dimension of $\mathcal{C}$ is denoted by $\operatorname{dim}(\mathcal{C})$, and the coordinates of $\mathcal{C}$ are indexed by the integers from the set $[n]=\{1,2, \ldots, n\}$ as usual. We will also associate with the coordinates of $\mathcal{C}$ a set, $E(\mathcal{C})$, of coordinate labels, so that there is a bijection $\alpha_{\mathcal{C}}:[n] \rightarrow E(\mathcal{C})$. The label sequence of $\mathcal{C}$ is defined to be the $n$-tuple $\left(\alpha_{1}, \alpha_{2}, \ldots, \alpha_{n}\right)$, where $\alpha_{i}=\alpha_{\mathcal{C}}(i)$. For notational convenience, we will simply let $\alpha_{\mathcal{C}}$ denote the label sequence of $\mathcal{C}$. Unless specified otherwise (as in the

\footnotetext{
${ }^{1}$ In this paper, we take the connectivity function of a matroid $M$ with ground set $E$ and rank function $r$ to be $\lambda_{M}(X)=r(X)+r(E-X)-r(E)$ for $X \subset E$. Therefore, what we consider to be matroids of pathwidth one would be matroids of pathwidth two in [7], [9].
} 
case of code minors and duals below), we will, by default, set $E(\mathcal{C})$ to be $[n]$, and $\alpha_{\mathcal{C}}$ to be the $n$-tuple $(1,2,3, \ldots, n)$. In such a case, the label of each coordinate is the same as its index.

Given a code $\mathcal{C}$ over $\mathbb{F}_{q}$, specified by a generator matrix $G$, we define its associated matroid $M(\mathcal{C})$ to be the vector matroid, $M[G]$, of $G$. We identify the ground set of $M(\mathcal{C})$ with $E(\mathcal{C})$. Note that if $G$ and $G^{\prime}$ are distinct generator matrices of the code $\mathcal{C}$, then $M[G]=M\left[G^{\prime}\right]$, and hence, $M(\mathcal{C})$ is independent of the choice of generator matrix. Thus, any generator matrix of $\mathcal{C}$ is an $\mathbb{F}_{q}$-representation of $M(\mathcal{C})$.

Conversely, if $M$ is an $\mathbb{F}_{q}$-representable matroid, and $G$ is an $\mathbb{F}_{q}$-representation of $M$, then $M=M(\mathcal{C})$ for the code $\mathcal{C}$ generated by $G$. Thus, each $\mathbb{F}_{q}$-representable matroid is associated with some code $\mathcal{C}$ over $\mathbb{F}_{q}$.

For any code $\mathcal{C}$, the dual code, $\mathcal{C}^{\perp}$, is specified to have the same label sequence as $\mathcal{C}$, i.e., $\alpha_{\mathcal{C}^{\perp}}=\alpha_{\mathcal{C}}$. It is a particularly nice fact [15, Theorem 2.2.8] that the matroids associated with $\mathcal{C}$ and $\mathcal{C}^{\perp}$ are dual to each other, i.e., $M\left(\mathcal{C}^{\perp}\right)=(M(\mathcal{C}))^{*} \stackrel{\text { def }}{=} M^{*}(\mathcal{C})$.

Given a $J \subset E(\mathcal{C})$, we will denote by $\mathcal{C} \backslash J$ (resp. $\mathcal{C} / J$ ) the code obtained from $\mathcal{C}$ by puncturing (resp. shortening at) those coordinates having labels in $J$. Thus, $\mathcal{C} / J=\left(\mathcal{C}^{\perp} \backslash J\right)^{\perp}$. A minor of $\mathcal{C}$ is a code of the form $\mathcal{C} / X \backslash Y$ for disjoint subsets $X, Y \subset E(\mathcal{C})$. A minor of $\mathcal{C}$ that is not $\mathcal{C}$ itself is called a proper minor of $\mathcal{C}$. The coordinates of a minor of $\mathcal{C}$ retain their labels from $E(\mathcal{C})$. More precisely, we set $E(\mathcal{C} / X \backslash Y)=E(\mathcal{C})-(X \cup Y)$, and take the label sequence of $\mathcal{C} / X \backslash Y$ to be the $(n-|X \cup Y|)$-tuple obtained from $\alpha_{\mathcal{C}}=\left(\alpha_{1}, \alpha_{2}, \ldots, \alpha_{n}\right)$ by simply removing those entries that are in $X \cup Y$. The operations of puncturing and shortening correspond to the matroid-theoretic operations of deletion and contraction, respectively: for $J \subset$ $E(\mathcal{C})$

$$
M(\mathcal{C} \backslash J)=M(\mathcal{C}) \backslash J \quad \text { and } \quad M(\mathcal{C} / J)=M(\mathcal{C}) / J .
$$

We will find it convenient to use $\left.\mathcal{C}\right|_{J}$ to denote the restriction of $\mathcal{C}$ to the coordinates with labels in $J$, i.e., $\left.\mathcal{C}\right|_{J}=\mathcal{C} \backslash J^{c}$, where $J^{c}$ denotes the set difference $E(\mathcal{C})-J$. This allows us to express the rank function, $r: E(\mathcal{C}) \rightarrow \mathbb{Z}$, of the matroid $M(\mathcal{C})$ as follows: for $J \subset E(\mathcal{C}), r(J)=\operatorname{dim}\left(\left.\mathcal{C}\right|_{J}\right)$.

Two length- $n$ linear codes $\mathcal{C}$ and $\mathcal{C}^{\prime}$ over $\mathbb{F}_{q}$ are defined to be equivalent if there is an $n \times n$ permutation matrix $\Pi$ and an invertible $n \times n$ diagonal matrix $\Delta$, such that $\mathcal{C}^{\prime}$ is the image of $\mathcal{C}$ under the vector space isomorphism $\phi: \mathbb{F}_{q}^{n} \rightarrow \mathbb{F}_{q}^{n}$ defined by $\phi(\mathbf{x})=(\Pi \Delta) \mathbf{x}$. Informally, $\mathcal{C}^{\prime}$ is equivalent to $\mathcal{C}$ if $\mathcal{C}^{\prime}$ can be obtained by first multiplying the coordinates of $\mathcal{C}$ by some nonzero elements of $\mathbb{F}_{q}$, and then applying a coordinate permutation. In such a case, we write $\mathcal{C} \equiv \mathcal{C}^{\prime}$. The equivalence class of codes equivalent to $\mathcal{C}$ will be denoted by $[\mathcal{C}]$. It is clear that if codes $\mathcal{C}$ and $\mathcal{C}^{\prime}$ are equivalent, then their associated matroids are isomorphic.

We remark that code equivalence has been defined above according to the codingtheoretic convention. Note that, under this definition, if $\mathcal{C}^{\prime}$ is obtained by applying an automorphism of the field $\mathbb{F}_{q}$ to $\mathcal{C}$, then $\mathcal{C}$ and $\mathcal{C}^{\prime}$ would in general be considered to be inequivalent.

A family, $\mathfrak{C}$, of codes over $\mathbb{F}_{q}$ is said to be minor-closed if, for each $\mathcal{C} \in \mathfrak{C}$, any code equivalent to a minor of $\mathcal{C}$ is also in $\mathfrak{C}$. A code, $\mathcal{D}$, over $\mathbb{F}_{q}$ is said to be an excluded minor for a minor-closed family $\mathfrak{C}$, if $\mathcal{D} \notin \mathfrak{C}$, but every proper minor of $\mathcal{D}$ is in $\mathfrak{C}$. It is easily verified that if $\mathfrak{C}$ is a minor-closed family, then a code $\mathcal{C}$ is in $\mathfrak{C}$ iff no minor of $\mathcal{C}$ is an excluded minor for $\mathfrak{C}$.

Given a collection, $\mathcal{M}$, of $\mathbb{F}_{q}$-representable matroids, define the code family

$$
\mathfrak{C}(\mathcal{M})=\left\{\mathcal{C}: \mathcal{C} \text { is a linear code over } \mathbb{F}_{q} \text { such that } M(\mathcal{C}) \in \mathcal{M}\right\} .
$$


Evidently, if $\mathcal{M}$ is a minor-closed class of $\mathbb{F}_{q}$-representable matroids, then $\mathfrak{C}(\mathcal{M})$ is also minor-closed. In this case, if $\mathcal{F}$ is the set of all excluded minors for $\mathcal{M}$, then $\mathfrak{C}(\mathcal{F})$ is the set of all excluded minors for $\mathfrak{C}(\mathcal{M})$.

2.2. Pathwidth, Trellis-width and Branchwidth. The definitions in this section rely on the notion of the connectivity function of a matroid. Let $M$ be a matroid with ground set $E(M)$ and rank function $r_{M}$. Its connectivity function, $\lambda_{M}$, is defined by $\lambda_{M}(X)=r_{M}(X)+r_{M}(E(M)-X)-r_{M}(E(M))$ for $X \subset E(M)$. Note that $\lambda_{M}(X)=\lambda_{M}(E(M)-X)$, and $\lambda_{M}(E(M))=\lambda_{M}(\emptyset)=0$. It should be pointed out that in the matroid theory literature, the prevalent definition of the connectivity function adds a ' +1 ' to the expression we have given. We have chosen not to follow suit in order that we can give a minimum-fuss definition of trellis-width below.

The connectivity function is non-negative, i.e., $\lambda_{M}(X) \geq 0$ for all $X \subset E(M)$, and submodular i.e., $\lambda_{M}(X \cup Y)+\lambda_{M}(X \cap Y) \leq \lambda_{M}(X)+\lambda_{M}(Y)$ for all $X, Y \subset E(M)$. It is monotone under the action of taking minors - if $N$ is a minor of $M$, then for all $X \subset E(N), \lambda_{N}(X) \leq \lambda_{M}(X)$. Finally, the connectivity function of a matroid is identical to that of its dual, i.e., $\lambda_{M}(X)=\lambda_{M^{*}}(X)$ for all $X \subset E(M)$.

Given an ordering $\left(e_{1}, e_{2}, \ldots, e_{n}\right)$ of the elements of $M$, define the width of the ordering to be $w_{M}\left(e_{1}, e_{2}, \ldots, e_{n}\right)=\max _{i \in[n]} \lambda_{M}\left(e_{1}, e_{2}, \ldots, e_{i}\right)$. (For simplicity of notation, we use $\lambda_{M}\left(e_{1}, e_{2}, \ldots, e_{i}\right)$ instead of $\lambda_{M}\left(\left\{e_{1}, e_{2}, \ldots, e_{i}\right\}\right)$.) The pathwidth of $M$ is defined as $\operatorname{pw}(M)=\min w_{M}\left(e_{1}, e_{2}, \ldots, e_{n}\right)$, the minimum being taken over all orderings $\left(e_{1}, e_{2}, \ldots, e_{n}\right)$ of $E(M)$. An ordering $\left(e_{1}, e_{2}, \ldots, e_{n}\right)$ of $E(M)$ such that $w_{M}\left(e_{1}, e_{2}, \ldots, e_{n}\right)=\operatorname{pw}(M)$ is called an optimal ordering.

Since $\lambda_{M} \equiv \lambda_{M^{*}}$, it is clear that $\operatorname{pw}(M)=\operatorname{pw}\left(M^{*}\right)$. Another useful and easily verifiable property of pathwidth is that, for matroids $M_{1}$ and $M_{2}$, the pathwidth of their direct sum, $\operatorname{pw}\left(M_{1} \oplus M_{2}\right)$, equals $\max \left\{\operatorname{pw}\left(M_{1}\right), \operatorname{pw}\left(M_{2}\right)\right\}$. The property of pathwidth most important for our purposes is stated in the following lemma.

Lemma 2.1. If $N$ is a minor of $M$, then $p w(N) \leq p w(M)$.

Proof. Let $\left(e_{1}, \ldots, e_{n}\right)$ be an optimal ordering of $E(M)$. It is enough to show the result in the case when $N=M \backslash e_{i}$ or $N=M / e_{i}$ for some $i \in[n]$. In such a case, consider the ordering $\left(e_{1}, \ldots, e_{i-1}, e_{i+1}, \ldots, e_{n}\right)$ of $E(N)$. For $j \in\{1, \ldots, i-1\}$, we have $\lambda_{N}\left(e_{1}, \ldots, e_{j}\right) \leq \lambda_{M}\left(e_{1}, \ldots, e_{j}\right)$. For $j \in\{i+1, \ldots, n\}$, we have

$$
\begin{aligned}
\lambda_{N}\left(e_{1}, \ldots, e_{i-1}, e_{i+1}, \ldots, e_{j}\right) & =\lambda_{N}\left(e_{j+1}, \ldots, e_{n}\right) \\
& \leq \lambda_{M}\left(e_{j+1}, \ldots, e_{n}\right)=\lambda_{M}\left(e_{1}, \ldots, e_{j}\right) .
\end{aligned}
$$

It follows that $w_{N}\left(e_{1}, \ldots, e_{i-1}, e_{i+1}, \ldots, e_{n}\right) \leq w_{M}\left(e_{1}, \ldots, e_{n}\right)=\operatorname{pw}(M)$, and hence, $\operatorname{pw}(N) \leq \operatorname{pw}(M)$.

The trellis-width of a linear code $\mathcal{C}$ over $\mathbb{F}_{q}$ is defined to be $\operatorname{tw}(\mathcal{C})=\operatorname{pw}(M(\mathcal{C}))$. For a discussion of the motivation and practical implications of this definition, we refer the reader to [17, Section 5].

The pathwidth of a matroid is an upper bound on its branchwidth, a more well known measure of matroid complexity. The branchwidth of a matroid is defined via cubic trees. A cubic tree is a tree in which the degree of any vertex is either one or three. The vertices of degree one are called leaves. A branch-decomposition of a matroid $M$ is a cubic tree, $T$, with $|E(M)|$ leaves, labelled in a one-to-one fashion by the elements of $M$. Each edge $e$ of such a branch-decomposition $T$ connects two subtrees of $T$, so $T \backslash e$ has two components. We say that edge $e$ displays a subset $X \subset E(M)$ if $X$ is the set of labels of leaves of one of the components of $T \backslash e$. The 


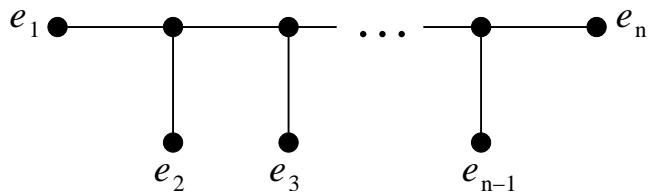

FIG. 2.1. A branch-decomposition of $M$ having width equal to $w_{M}\left(e_{1}, e_{2}, \ldots, e_{n}\right)$.

width of an edge $e$ of $T$ is defined to be $\lambda_{M}(X)$, where $X$ is one of the label sets displayed by $e$. The width of $T$ is the maximum among the widths of its edges.

The branchwidth of $M$ is the minimum among the widths of all its branchdecompositions. Note that if $T$ is the branch-decomposition of $M$ shown in Figure 2.1, then the width of $T$ is precisely $w_{M}\left(e_{1}, e_{2}, \ldots, e_{n}\right)$. Indeed, the width of any edge of $T$ is either $\lambda_{M}\left(e_{i}\right)$ or $\lambda_{M}\left(e_{1}, \ldots, e_{i}\right)$ for some $i \in[n]$. Now, for any $i \in[n]$,

$$
\begin{aligned}
\lambda_{M}\left(e_{1}, \ldots, e_{i-1}\right)+\lambda_{M}\left(e_{1}, \ldots, e_{i}\right) & =\lambda_{M}\left(e_{i}, e_{i+1}, \ldots, e_{n}\right)+\lambda_{M}\left(e_{1}, \ldots, e_{i}\right) \\
& \geq \lambda_{M}(E(M))+\lambda_{M}\left(e_{i}\right)=\lambda_{M}\left(e_{i}\right),
\end{aligned}
$$

the inequality above arising from the submodularity of $\lambda_{M}$. Since $\lambda_{M}\left(e_{i}\right) \in\{0,1\}$, either $\lambda_{M}\left(e_{1}, \ldots, e_{i-1}\right)$ or $\lambda_{M}\left(e_{1}, \ldots, e_{i}\right)$ is at least as large as $\lambda_{M}\left(e_{i}\right)$. Therefore, the width of $T$ is given by $\max _{i \in[n]} \lambda_{M}\left(e_{1}, \ldots, e_{i}\right)=w_{M}\left(e_{1}, \ldots, e_{n}\right)$. It follows that the branchwidth of $M$ is upper-bounded by $\operatorname{pw}(M)$.

3. NP-Hardness of Matroid Pathwidth and Code Trellis-Width. In this section, we prove that for any fixed field $\mathbb{F}$, the problem of computing the pathwidth of an $\mathbb{F}$-representable matroid $M$, given a representation of $M$ over $\mathbb{F}$, is NP-hard. We accomplish this by reduction from the known NP-hard problem of computing the pathwidth of a graph [1, 2].

The notion of graph pathwidth was introduced by Robertson and Seymour in [16. Let $\mathcal{G}$ be a graph with vertex set $V$. An ordered collection $\mathcal{V}=\left(V_{1}, \ldots, V_{t}\right)$, $t \geq 1$, of subsets of $V$ is called a path-decomposition of $\mathcal{G}$, if

(i) $\bigcup_{i=1}^{t} V_{i}=V$;

(ii) for each pair of adjacent vertices $u, v \in V$, we have $\{u, v\} \subset V_{i}$ for some $i \in[t]$; and

(iii) for $1 \leq i<j<k \leq t, V_{i} \cap V_{k} \subset V_{j}$.

The width of such a path-decomposition $\mathcal{V}$ is defined to be $w_{\mathcal{G}}(\mathcal{V})=\max _{i \in[t]}\left|V_{i}\right|-1$. The pathwidth of $\mathcal{G}$, denoted by $\operatorname{pw}(\mathcal{G})$, is the minimum among the widths of all its path-decompositions. A path-decomposition $\mathcal{V}$ such that $w_{\mathcal{G}}(\mathcal{V})=\mathrm{pw}(\mathcal{G})$ is called an optimal path-decomposition of $\mathcal{G}$.

Let $\mathbb{F}$ be an arbitrary field. Given a graph $\mathcal{G}$ with vertex set $V$, our aim is to produce, in time polynomial in $|V|$, a matrix $A$ over $\mathbb{F}$ such that pw $(\mathcal{G})$ can be directly computed from pw $(M[A])$. The NP-hardness of computing graph pathwidth then implies the NP-hardness of computing the pathwidth of an $\mathbb{F}$-representable matroid.

The obvious idea of taking $A$ to be a representation of the cycle matroid of $\mathcal{G}$ does not work. As observed by Robertson and Seymour [16], trees can have arbitrarily large pathwidth; however, the cycle matroid of any tree is $U_{n, n}$ for some $n$, and $\operatorname{pw}\left(U_{n, n}\right)=0$. What actually turns out to work is to take $A$ to be a representation of the cycle matroid of a certain graph constructible from $\mathcal{G}$ in polynomial time, as we describe next.

Let $\mathcal{G}^{\prime}$ be a graph defined on the same vertex set, $V$, as $\mathcal{G}$, having the following properties (see Figure 3.1): 


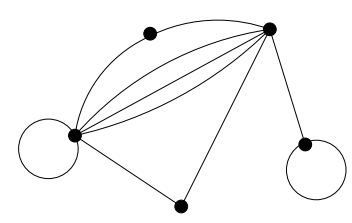

$G$

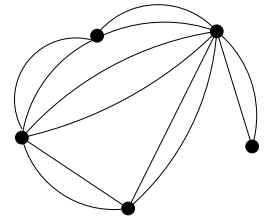

$G^{\prime}$

FIG. 3.1. Construction of $\mathcal{G}^{\prime}$ from $\mathcal{G}$.

(P1) $\mathcal{G}^{\prime}$ is loopless;

(P2) a pair of distinct vertices is adjacent in $\mathcal{G}^{\prime}$ iff it is adjacent in $\mathcal{G}$; and

(P3) in $\mathcal{G}^{\prime}$, there are exactly two edges between each pair of adjacent vertices.

It is evident from the definition that $\left(V_{1}, \ldots, V_{t}\right)$ is a path-decomposition of $\mathcal{G}$ iff it is a path-decomposition of $\mathcal{G}^{\prime}$. Therefore, $\operatorname{pw}\left(\mathcal{G}^{\prime}\right)=\operatorname{pw}(\mathcal{G})$.

Define $\overline{\mathcal{G}}$ to be the graph obtained by adding an extra vertex, henceforth denoted by $x$, to $\mathcal{G}^{\prime}$, along with a pair of parallel edges from $x$ to each $v \in V$ (see Figure 3.2). Clearly, $\overline{\mathcal{G}}$ is constructible directly from $\mathcal{G}$ in $O\left(|V|^{2}\right)$ time. But more importantly, the pathwidth of the cycle matroid, $M(\overline{\mathcal{G}})$, of $\overline{\mathcal{G}}$ relates very simply to the pathwidth of $\mathcal{G}$, as made precise by the following proposition.

Proposition 3.1. $p w(M(\overline{\mathcal{G}}))=p w(\mathcal{G})+1$.

Before proving the result, we present some of its implications. For any field $\mathbb{F}$, $M(\overline{\mathcal{G}})$ is $\mathbb{F}$-representable. Indeed, if $D(\overline{\mathcal{G}})$ is any directed graph obtained by arbitrarily assigning orientations to the edges of $\overline{\mathcal{G}}$, then the vertex-arc incidence matrix of $D(\overline{\mathcal{G}})$ is an $\mathbb{F}$-representation of $M(\overline{\mathcal{G}})[15$, Proposition 5.1.2]. It is easily verified that such an $\mathbb{F}$-representation of $M(\overline{\mathcal{G}})$ can be constructed directly from $\mathcal{G}$ in $O\left(|V|^{3}\right)$ time. Now, suppose that there were a polynomial-time algorithm for computing the pathwidth of an arbitrary $\mathbb{F}$-representable matroid, given an $\mathbb{F}$-representation for it. Then, given any graph $\mathcal{G}$, we can construct an $\mathbb{F}$-representation, $A$, of $M(\overline{\mathcal{G}})$, and then compute the pathwidth of $M[A]=M(\overline{\mathcal{G}})$, all in polynomial time. Therefore, by Proposition 3.1, we have a polynomial-time algorithm to compute the pathwidth of $\mathcal{G}$. However, the graph pathwidth problem is NP-hard. So, if there exists a polynomial-time algorithm for it, then we must have $P=N P$. This implies the following result.

TheOREM 3.2. Let $\mathbb{F}$ be a fixed field. The problem of computing the pathwidth of $M[A]$, for an arbitrary matrix $A$ over $\mathbb{F}$, is NP-hard.

As a corollary, we have that computing the trellis-width of a code is NP-hard.

Corollary 3.3. Let $\mathbb{F}$ be a fixed finite field. The problem of computing the trelliswidth of an arbitrary linear code over $\mathbb{F}$, specified by any of its generator matrices, is NP-hard.

The remainder of this section is devoted to the proof of Proposition 3.1. Since $\operatorname{pw}\left(\mathcal{G}^{\prime}\right)=\operatorname{pw}(\mathcal{G})$, for the purpose of our proof, we may assume that $\mathcal{G}^{\prime}=\mathcal{G}$. Thus, from now until the end of this section, we take $\mathcal{G}$ to be a loopless graph satisfying property (P3) above. Note that $\overline{\mathcal{G}}$ also satisfies (P3). For each pair of adjacent vertices $u, v$ in $\mathcal{G}$ or $\overline{\mathcal{G}}$, we denote by $l_{u v}$ and $r_{u v}$ the two edges between $u$ and $v$. Let $V$ and $E$ denote the sets of vertices and edges of $\mathcal{G}$, and let $\bar{V}$ and $\bar{E}$ denote the corresponding sets of $\overline{\mathcal{G}}$. We thus have $\bar{V}=V \dot{\cup}\{x\}$, and $\bar{E}=E \dot{\cup}\left(\bigcup_{v \in V}\left\{l_{x v}, r_{x v}\right\}\right)$. 


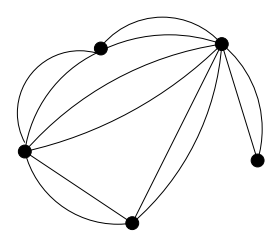

$G^{\prime}$

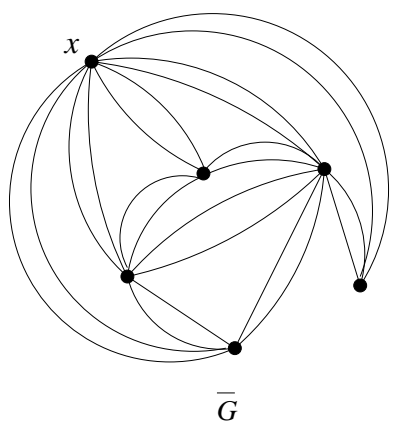

Fig. 3.2. Construction of $\overline{\mathcal{G}}$ from $\mathcal{G}^{\prime}$.

Set $M=M(\overline{\mathcal{G}})$, so that $E(M)=\bar{E}$. Note that since $\overline{\mathcal{G}}$ is connected (each $v \in V$ is adjacent to $x)$, we have $\operatorname{rank}(M)=|\bar{V}|-1=|V|$.

We will first prove that $\operatorname{pw}(M) \leq \operatorname{pw}(\mathcal{G})+1$. Let $\mathcal{V}=\left(V_{1}, \ldots, V_{t}\right)$ be a pathdecomposition of $\mathcal{G}$. We need the following fact about $\mathcal{V}$ : for each $j \in[t]$,

$$
\bigcup_{i \leq j} V_{i} \cap \bigcup_{k \geq j} V_{k}=V_{j}
$$

The above equality follows from the fact that a path-decomposition, by definition, has the property that for $1 \leq i<j<k \leq t, V_{i} \cap V_{k} \subset V_{j}$.

For $j \in[t]$, let $F_{j}$ be the set of edges of $\mathcal{G}$ that have both their end-points in $V_{j}$. By condition (ii) in the definition of path-decomposition, $\bigcup_{j=1}^{t} F_{j}=E$. Now, let $\overline{F_{j}}=F_{j} \cup\left(\bigcup_{v \in V_{j}}\left\{l_{x v}, r_{x v}\right\}\right)$, so that $\bigcup_{j=1}^{t} \overline{F_{j}}=\bar{E}$.

Definition 3.1. An ordering $\left(e_{1}, \ldots, e_{n}\right)$ of the elements of a matroid $M$ is said to induce an ordered partition $\left(E_{1}, \ldots, E_{t}\right)$ of $E(M)$ if for each $j \in[t],\left\{e_{n_{j-1}+1}\right.$, $\left.e_{n_{j-1}+2}, \ldots, e_{n_{j}}\right\}=E_{j}$, where $n_{j}=\left|\bigcup_{i \leq j} E_{j}\right|\left(\right.$ and $\left.n_{0}=0\right)$.

Let $\pi=\left(e_{1}, \ldots, e_{n}\right)$ be any ordering of $\bar{E}$ that induces the ordered partition $\left(E_{1}, E_{2}, \ldots, E_{t}\right)$, where for each $j \in[t], E_{j}=\overline{F_{j}}-\bigcup_{i<j} \overline{F_{i}}$. We claim that the width of $\pi$ is at most one more than the width of the path-decomposition $\mathcal{V}$.

LEMMA 3.4. $w_{M}(\pi) \leq w_{\mathcal{G}}(\mathcal{V})+1$.

Proof. Observe first that

$$
\begin{aligned}
w_{M}(\pi) & =\max _{j \in[t]} \max _{1 \leq k \leq n_{j}-n_{j-1}} \lambda_{M}\left(\bigcup_{i<j} E_{i} \cup\left\{e_{n_{j-1}+1}, \ldots, e_{n_{j-1}+k}\right\}\right) \\
& \leq \max _{j \in[t]} \max _{E^{\prime} \subset E_{j}} \lambda_{M}\left(\bigcup_{i<j} E_{i} \cup E^{\prime}\right) .
\end{aligned}
$$

Let $X=\bigcup_{i<j} E_{i} \cup E^{\prime}$ for some $j \in[t]$ and $E^{\prime} \subset E_{j}$, and consider $\lambda_{M}(X)=$ $r_{M}(X)+r_{M}(\bar{E}-X)-r_{M}(\bar{E})$. Since $\overline{\mathcal{G}}$ is a connected graph, $r_{M}(\bar{E})=|\bar{V}|-1=|V|$.

If $v$ is a vertex of $\overline{\mathcal{G}}$ incident with an edge in $X$, then $v \in \bigcup_{i \leq j} V_{j} \dot{\cup}\{x\}$. So, the subgraph of $\overline{\mathcal{G}}$ induced by $X$ has its vertices contained in $\bigcup_{i \leq j} V_{j} \dot{\cup}\{x\}$. Therefore, $r_{M}(X) \leq\left|\bigcup_{i \leq j} V_{j} \dot{\cup}\{x\}\right|-1=\left|\bigcup_{i \leq j} V_{j}\right|$. 
Next, consider $\bar{E}-X=\left(\bigcup_{k>j} E_{k}\right) \cup\left(E_{j}-E^{\prime}\right)$. Reasoning as above, the subgraph of $\mathcal{G}$ induced by $\bar{E}-X$ has its vertices contained in $\bigcup_{k \geq j} V_{k} \dot{\cup}\{x\}$. Hence, $r_{M}(\bar{E}-$ $X) \leq\left|\bigcup_{k \geq j} V_{k}\right|$.

Therefore, we have

$$
\begin{aligned}
\lambda_{M}(X) & \leq\left|\bigcup_{i \leq j} V_{j}\right|+\left|\bigcup_{k \geq j} V_{k}\right|-|V| \\
& =\left|\bigcup_{i \leq j} V_{j} \cap \bigcup_{k \geq j} V_{k}\right|=\left|V_{j}\right|,
\end{aligned}
$$

the last equality arising from (3.1). Hence, carrying on from (3.2),

$$
w_{M}(\pi) \leq \max _{j \in[t]}\left|V_{j}\right|=w_{\mathcal{G}}(\mathcal{V})+1,
$$

as desired. $\square$

The fact that $\operatorname{pw}(M) \leq \operatorname{pw}(\mathcal{G})+1$ easily follows from the above lemma. Indeed, we may choose $\mathcal{V}$ to be an optimal path-decomposition of $\mathcal{G}$. Then, by Lemma 3.4. there exists an ordering $\left(e_{1}, \ldots, e_{n}\right)$ of $E(M)$ such that $w_{M}\left(e_{1}, \ldots, e_{n}\right) \leq \operatorname{pw}(\mathcal{G})+1$. Hence, $\operatorname{pw}(M) \leq w_{M}\left(e_{1}, \ldots, e_{n}\right) \leq \operatorname{pw}(\mathcal{G})+1$.

We prove the reverse inequality in two steps, first showing that $\mathrm{pw}(\overline{\mathcal{G}})=\operatorname{pw}(\mathcal{G})+1$, and then showing that $\mathrm{pw}(M) \geq \mathrm{pw}(\overline{\mathcal{G}})$.

Lemma 3.5. $p w(\overline{\mathcal{G}})=p w(\mathcal{G})+1$.

Proof. Clearly, if $\mathcal{V}=\left(V_{1}, \ldots, V_{t}\right)$ is a path-decomposition of $\mathcal{G}$, then $\overline{\mathcal{V}}=\left(V_{1} \cup\right.$ $\left.\{x\}, \ldots, V_{t} \cup\{x\}\right)$ is a path-decomposition of $\overline{\mathcal{G}}$. Hence, choosing $\mathcal{V}$ to be an optimal path-decomposition of $\mathcal{G}$, we have that $\operatorname{pw}(\overline{\mathcal{G}}) \leq w_{\overline{\mathcal{G}}}(\overline{\mathcal{V}})=w_{\mathcal{G}}(\mathcal{V})+1=\operatorname{pw}(\mathcal{G})+1$.

For the inequality in the other direction, we will show that there exists an optimal path-decomposition, $\widetilde{\mathcal{V}}=\left(\widetilde{V}_{1}, \ldots, \widetilde{V}_{s}\right)$, of $\overline{\mathcal{G}}$ such that $x \in \widetilde{V}_{i}$ for all $i \in[s]$. We then have $\mathcal{V}=\left(\widetilde{V}_{1}-\{x\}, \ldots, \widetilde{V}_{s}-\{x\}\right)$ being a path-decomposition of $\mathcal{G}$, and hence, $\operatorname{pw}(\mathcal{G}) \leq w_{\mathcal{G}}(\mathcal{V})=w_{\overline{\mathcal{G}}}(\widetilde{\mathcal{V}})-1=\operatorname{pw}(\overline{\mathcal{G}})-1$.

Let $\overline{\mathcal{V}}=\left(\bar{V}_{1}, \ldots, \bar{V}_{t}\right)$ be an optimal path-decomposition of $\overline{\mathcal{G}}$, and let $i_{0}=\min \{i$ : $\left.x \in \bar{V}_{i}\right\}$ and $i_{1}=\max \left\{i: x \in \bar{V}_{i}\right\}$. Since $\bar{V}_{i} \cap \bar{V}_{k} \subset \bar{V}_{j}$ for $i<j<k$, we must have $x \in \bar{V}_{i}$ for each $i \in\left[i_{0}, i_{1}\right]$.

We claim that $\left(\bar{V}_{i_{0}}, \bar{V}_{i_{0}+1}, \ldots, \bar{V}_{i_{1}}\right)$ is a path-decomposition of $\overline{\mathcal{G}}$. We only have to show that $\bigcup_{i=i_{0}}^{i_{1}} \bar{V}_{i}=\bar{V}$, and that for each pair of adjacent vertices $u, v \in \bar{V}$, $\{u, v\} \subset \bar{V}_{i}$ for some $i \in\left[i_{0}, i_{1}\right]$. To see why the first assertion is true, consider any $v \in \bar{V}, v \neq x$. Since $x$ is adjacent to $v$, and $\overline{\mathcal{V}}$ is a path-decomposition of $\overline{\mathcal{G}}$, $\{x, v\} \subset \bar{V}_{i}$ for some $i \in[t]$. However, $x \in \bar{V}_{i}$ iff $i \in\left[i_{0}, i_{1}\right]$, and so, $\{x, v\} \subset \bar{V}_{i}$ for some $i \in\left[i_{0}, i_{1}\right]$. In particular, $v \in \bar{V}_{i}$ for some $i \in\left[i_{0}, i_{1}\right]$.

For the second assertion, suppose that $u, v$ is a pair of vertices adjacent in $\overline{\mathcal{G}}$. Obviously, $\{u, v\} \subset \bar{V}_{j}$ for some $j \in[t]$. Suppose that $j \notin\left[i_{0}, i_{1}\right]$. We consider the case when $j>i_{1}$; the case when $j<i_{0}$ is similar. As $\bigcup_{i=i_{0}}^{i_{1}} \bar{V}_{i}=\bar{V}$, there exist $i_{2}, i_{3} \in\left[i_{0}, i_{1}\right]$ such that $u \in \bar{V}_{i_{2}}$ and $v \in \bar{V}_{i_{3}}$. Without loss of generality (WLOG), $i_{2} \leq i_{3}$. If $i_{2}=i_{3}$, then there exists $i \in\left[i_{0}, i_{1}\right]$ such that $\{u, v\} \subset \bar{V}_{i}$. If $i_{2}<i_{3}$, we 
have $u \in \bar{V}_{i_{2}} \cap \bar{V}_{j}$ and $i_{2}<i_{3}<j$. Hence, $u \in \bar{V}_{i_{3}}$ as well, and so once again, we have an $i \in\left[i_{0}, i_{1}\right]$ such that $\{u, v\} \in \bar{V}_{i}$.

Thus, $\left(\bar{V}_{i_{0}}, \bar{V}_{i_{0}+1}, \ldots, \bar{V}_{i_{1}}\right)$ is a path-decomposition of $\overline{\mathcal{G}}$, with the property that $x \in \bar{V}_{i}$ for all $i \in\left[i_{0}, i_{1}\right]$. It must be an optimal path-decomposition, since it is a subsequence of the optimal path-decomposition $\overline{\mathcal{V}}$.

To complete the proof of Proposition 3.1 it remains to show that $\mathrm{pw}(M) \geq \mathrm{pw}(\overline{\mathcal{G}})$. We introduce some notation at this point. Recall that the two edges between a pair of adjacent vertices $u$ and $v$ in $\overline{\mathcal{G}}$ (or $\mathcal{G}$ ) are denoted by $l_{u v}$ and $r_{u v}$. We define

$$
\begin{aligned}
L_{\mathcal{G}} & =\left\{l_{u v}: u, v \text { are adjacent vertices in } \mathcal{G}\right\} \\
R_{\mathcal{G}} & =\left\{r_{u v}: u, v \text { are adjacent vertices in } \mathcal{G}\right\}
\end{aligned}
$$

$L_{x}=\bigcup_{v \in V}\left\{l_{x v}\right\}$ and $R_{x}=\bigcup_{v \in V}\left\{r_{x v}\right\}$, where $x$ is the distinguished vertex in $\bar{V}-V$. Thus, $L_{\mathcal{G}} \cup R_{\mathcal{G}}=E$ and $E \cup L_{x} \cup R_{x}=\bar{E}$. Note that, by construction of $\overline{\mathcal{G}}, \operatorname{cl}_{M}\left(L_{x}\right)=$ $\operatorname{cl}_{M}\left(R_{x}\right)=\bar{E}$, where $\operatorname{cl}_{M}$ denotes the closure operator of $M$.

We will need the fact that there exists an optimal ordering $\left(e_{1}, \ldots, e_{n}\right)$ of $\bar{E}$ that induces a certain ordered partition of $\bar{E}$ of the form

$$
\left(L_{1}, A_{1}, B_{1}, R_{1}, L_{2}, A_{2}, B_{2}, R_{2}, \ldots, L_{t}, A_{t}, B_{t}, R_{t}\right)
$$

where for each $j \in[t], L_{j} \subset L_{x}, A_{j} \subset L_{\mathcal{G}}, B_{j} \subset R_{\mathcal{G}}$, and $R_{j} \subset R_{x}$. This will follow from a re-ordering argument given further below. But first, we make some simple observations about orderings of $\bar{E}$. Given an ordering of $\bar{E}$, we may assume, WLOG, that for each pair of adjacent vertices $u, v \in \bar{V}, l_{u v}$ appears before $r_{u v}$ in the ordering; we denote this by $l_{u v}<r_{u v}$. We call such an ordering of $\bar{E}$ a normal ordering.

Lemma 3.6. Let $\left(e_{1}, \ldots, e_{n}\right)$ be a normal ordering of $\bar{E}$. Then, for $1 \leq j \leq n-1$, we have

(a) $\lambda_{M}\left(e_{1}, \ldots, e_{j+1}\right)=\lambda_{M}\left(e_{1}, \ldots, e_{j}\right)+1$ iff $e_{j+1} \notin c l_{M}\left(e_{1}, \ldots, e_{j}\right)$; and

(b) $\lambda_{M}\left(e_{1}, \ldots, e_{j+1}\right)=\lambda_{M}\left(e_{1}, \ldots, e_{j}\right)-1$ iff $e_{j+1} \notin c l_{M}\left(e_{j+2}, \ldots, e_{n}\right)$.

Proof. We only prove (a), as the proof of (b) is similar. It is easy to deduce from the definition of the connectivity function that $\lambda_{M}\left(e_{1}, \ldots, e_{j+1}\right)=\lambda_{M}\left(e_{1}, \ldots, e_{j}\right)+1$ iff $e_{j+1} \notin \operatorname{cl}_{M}\left(e_{1}, \ldots, e_{j}\right)$ and $e_{j+1} \in \operatorname{cl}_{M}\left(e_{j+2}, \ldots, e_{n}\right)$.

Now, if $e_{j+1} \notin \mathrm{cl}_{M}\left(e_{1}, \ldots, e_{j}\right)$, then $e_{j+1}=l_{u v}$ for some $u, v$. (If not, i.e., if $e_{j+1}=r_{u v}$, then since $l_{u v}<r_{u v}$, we must have $l_{u v} \in\left\{e_{1}, \ldots, e_{j}\right\}$, and so, $e_{j+1}=r_{u v} \in \operatorname{cl}_{M}\left(l_{u v}\right) \subset \operatorname{cl}_{M}\left(e_{1}, \ldots, e_{j}\right)$, a contradiction.) Therefore, $\left\{e_{j+2}, \ldots, e_{n}\right\}$ contains $r_{u v}$, and hence, $e_{j+1}=l_{u v} \in \operatorname{cl}_{M}\left(e_{j+2}, \ldots, e_{n}\right)$. We have thus shown that if $e_{j+1} \notin \operatorname{cl}_{M}\left(e_{1}, \ldots, e_{j}\right)$, then $e_{j+1} \in \operatorname{cl}_{M}\left(e_{j+2}, \ldots, e_{n}\right)$. Part (a) of the lemma now follows.

We now describe a procedure that takes as input a normal ordering of $\bar{E}$, and produces as output a re-ordering of $\bar{E}$ with certain desirable properties. 


\section{$\underline{\text { RE-ORDERING ALGORITHM }}$}

Input: a normal ordering $\left(e_{1}, \ldots, e_{n}\right)$ of $\bar{E}$.

Initialization: $j=0$.

Step 0: If $j=0$, set $X_{j}=\emptyset$;

else, set $X_{j}=\operatorname{cl}_{M}\left(e_{1}, \ldots, e_{j}\right)-\left\{e_{1}, \ldots, e_{j}\right\}$.

Step 1: If $X_{j}=\emptyset$,

find the least $k>j$ such that

for some $m>j, e_{m} \in L_{x} \cap \mathrm{cl}_{M}\left(e_{1}, \ldots, e_{k}\right)$;

set $\left(e_{1}^{\prime}, \ldots, e_{n}^{\prime}\right)=\left(e_{1}, \ldots, e_{j}, e_{m}, e_{j+1}, \ldots, e_{m-1}, e_{m+1}, \ldots, e_{n}\right)$.

If $X_{j} \neq \emptyset$,

if $L_{x} \cap X_{j} \neq \emptyset$, find an $m>j$ such that $e_{m} \in L_{x} \cap X_{j}$;

else, if $L_{\mathcal{G}} \cap X_{j} \neq \emptyset$, find an $m>j$ such that $e_{m} \in L_{\mathcal{G}} \cap X_{j}$;

else, if $R_{\mathcal{G}} \cap X_{j} \neq \emptyset$, find an $m>j$ such that $e_{m} \in R_{\mathcal{G}} \cap X_{j}$;

else, if $R_{x} \cap X_{j} \neq \emptyset$, find an $m>j$ such that $e_{m} \in R_{x} \cap X_{j}$;

set $\left(e_{1}^{\prime}, \ldots, e_{n}^{\prime}\right)=\left(e_{1}, \ldots, e_{j}, e_{m}, e_{j+1}, \ldots, e_{m-1}, e_{m+1}, \ldots, e_{n}\right)$.

Step 2: $\quad$ Replace $j$ by $j+1$.

If $j<n$, replace $\left(e_{1}, \ldots, e_{n}\right)$ by $\left(e_{1}^{\prime}, \ldots, e_{n}^{\prime}\right)$, and return to Step 0;

else, output $\left(e_{1}^{\prime}, \ldots, e_{n}^{\prime}\right)$.

Denote by $\left(e_{1}^{*}, \ldots, e_{n}^{*}\right)$ the final output generated by the above algorithm. Set $X_{0}^{*}=\emptyset$, and for $j \in[n], X_{j}^{*}=\operatorname{cl}_{M}\left(e_{1}^{*}, \ldots, e_{j}^{*}\right)-\left\{e_{1}^{*}, \ldots, e_{j}^{*}\right\}$. Stepping through the algorithm, one may easily check that $\left(e_{1}^{*}, \ldots, e_{n}^{*}\right)$ has the following property: for $0 \leq j \leq n-1$, if $X_{j}^{*}=\emptyset$, then $e_{j+1}^{*} \in L_{x}$, and if $X_{j}^{*} \neq \emptyset$, then

$$
e_{j+1}^{*} \in \begin{cases}L_{x} \cap X_{j}^{*}, & \text { if } L_{x} \cap X_{j}^{*} \neq \emptyset \\ L_{\mathcal{G}} \cap X_{j}^{*}, & \text { if } L_{x} \cap X_{j}^{*}=\emptyset, \text { but } L_{\mathcal{G}} \cap X_{j}^{*} \neq \emptyset \\ R_{\mathcal{G}} \cap X_{j}^{*}, & \text { if } L_{x} \cap X_{j}^{*}=L_{\mathcal{G}} \cap X_{j}^{*}=\emptyset, \text { but } R_{\mathcal{G}} \cap X_{j}^{*} \neq \emptyset \\ R_{x} \cap X_{j}^{*}, & \text { if } L_{x} \cap X_{j}^{*}=L_{\mathcal{G}} \cap X_{j}^{*}=R_{\mathcal{G}} \cap X_{j}^{*}=\emptyset, \text { but } R_{x} \cap X_{j}^{*} \neq \emptyset .\end{cases}
$$

The following claim can be readily deduced from this property, and we leave the details to the reader.

CLAIM 3.7. (a) The ordering $\left(e_{1}^{*}, \ldots, e_{n}^{*}\right)$ induces an ordered partition of $\bar{E}$ of the form

$$
\left(L_{1}, A_{1}, B_{1}, R_{1}, L_{2}, A_{2}, B_{2}, R_{2}, \ldots, L_{t}, A_{t}, B_{t}, R_{t}\right),
$$

where for each $j \in[t], L_{j} \subset L_{x}, A_{j} \subset L_{\mathcal{G}}, B_{j} \subset R_{\mathcal{G}}$ and $R_{j} \subset R_{x}$. Moreover, for each $u, v \in \bar{V}, l_{u v} \in L_{j} \cup A_{j}$ iff $r_{u v} \in B_{j} \cup R_{j}$.

(b) For the ordered partition in (a), we have for each $j \in[t]$,

$$
A_{j} \cup B_{j} \subset c l_{M}\left(\bigcup_{i \leq j} L_{i}\right)-c l_{M}\left(\bigcup_{i<j} L_{i}\right) .
$$

The crucial property of $\left(e_{1}^{*}, \ldots, e_{n}^{*}\right)$ is the following. 
Lemma 3.8. If $\left(e_{1}^{*}, \ldots, e_{n}^{*}\right)$ is the output of the Re-ordering Algorithm in response to the input $\left(e_{1}, \ldots, e_{n}\right)$, then $w_{M}\left(e_{1}^{*}, \ldots, e_{n}^{*}\right) \leq w_{M}\left(e_{1}, \ldots, e_{n}\right)$.

Proof. Steps $0-1$ of the algorithm go through $n$ iterations, indexed by $j \in$ $\{0,1, \ldots, n-1\}$. In the $j$ th iteration, Step 1 is given a normal ordering $\left(e_{1}, \ldots, e_{n}\right)$, in response to which it produces an ordering $\left(e_{1}^{\prime}, \ldots, e_{n}^{\prime}\right)$, which is also normal. To prove the lemma, it is enough to show that $w_{M}\left(e_{1}^{\prime}, \ldots, e_{n}^{\prime}\right) \leq w_{M}\left(e_{1}, \ldots, e_{n}\right)$.

So, suppose that the algorithm is in its $j$ th iteration $(0 \leq j \leq n-1)$. We first dispose of the case when $X_{j} \neq \emptyset$. Then,

$$
\left(e_{1}^{\prime}, \ldots, e_{n}^{\prime}\right)=\left(e_{1}, \ldots, e_{j}, e_{m}, e_{j+1}, \ldots, e_{m-1}, e_{m+1}, \ldots, e_{n}\right)
$$

for some $m>j$ such that $e_{m} \in X_{j}$. Observe that if $1 \leq s \leq j$ or if $m \leq s \leq$ $n$, then $\left(e_{1}^{\prime}, \ldots, e_{s}^{\prime}\right)$ is just a re-ordering of $\left(e_{1}, \ldots, e_{s}\right)$, and hence $\lambda_{M}\left(e_{1}^{\prime}, \ldots, e_{s}^{\prime}\right)=$ $\lambda_{M}\left(e_{1}, \ldots, e_{s}\right)$.

So, consider $j<s<m$. In this case, $\left(e_{1}^{\prime}, \ldots, e_{s}^{\prime}\right)=\left(e_{1}, \ldots, e_{j}, e_{m}, e_{j+1}, \ldots, e_{s-1}\right)$. Since $e_{m} \in \operatorname{cl}_{M}\left(e_{1}, \ldots, e_{j}\right)$, we have $r_{M}\left(e_{1}^{\prime}, \ldots, e_{s}^{\prime}\right)=r_{M}\left(e_{1}, \ldots, e_{j}, e_{j+1}, \ldots, e_{s-1}\right)$. On the other hand,

$$
\begin{aligned}
r_{M}\left(e_{s+1}^{\prime}, \ldots, e_{n}^{\prime}\right) & =r_{M}\left(e_{s}, \ldots, e_{m-1}, e_{m+1}, \ldots, e_{n}\right) \\
& \leq r_{M}\left(e_{s}, \ldots, e_{m-1}, e_{m}, e_{m+1}, \ldots, e_{n}\right) .
\end{aligned}
$$

Hence, $\lambda\left(e_{1}^{\prime}, \ldots, e_{s}^{\prime}\right) \leq \lambda\left(e_{1}, \ldots, e_{s-1}\right)$. Therefore, for any $s \in[n]$, we have shown that there exists a $t \in[n]$ such that $\lambda\left(e_{1}^{\prime}, \ldots, e_{s}^{\prime}\right) \leq \lambda\left(e_{1}, \ldots, e_{t}\right)$. It follows that $w_{M}\left(e_{1}^{\prime}, \ldots, e_{n}^{\prime}\right) \leq w_{M}\left(e_{1}, \ldots, e_{n}\right)$.

We must now deal with the case when $X_{j}=\emptyset$, i.e., $\operatorname{cl}_{M}\left(e_{1}, \ldots, e_{j}\right)=\left\{e_{1}, \ldots, e_{j}\right\}$. Note that if $L_{x} \subset\left\{e_{1}, \ldots, e_{j}\right\}$, then since $\operatorname{cl}_{M}\left(L_{x}\right)=\bar{E}$, we have $\operatorname{cl}_{M}\left(e_{1}, \ldots, e_{j}\right)=\bar{E}$. Therefore, $\left\{e_{1}, \ldots, e_{j}\right\}=\bar{E}$, which means that $j=n$, a contradiction. Therefore, there must exist some $m>j$ such that $e_{m} \in L_{x}$.

Let $k^{*}$ be the least integer $k>j$ such that there exists $e_{m} \in L_{x} \cap \operatorname{cl}_{M}\left(e_{1}, \ldots, e_{k}\right)$ for some $m>j$. By choice of $k^{*}$, we have $m \geq k^{*}$. For this $m$, we again have

$$
\left(e_{1}^{\prime}, \ldots, e_{n}^{\prime}\right)=\left(e_{1}, \ldots, e_{j}, e_{m}, e_{j+1}, \ldots, e_{m-1}, e_{m+1}, \ldots, e_{n}\right) .
$$

As before, if $1 \leq s \leq j$ or if $m \leq s \leq n$, then $\lambda_{M}\left(e_{1}^{\prime}, \ldots, e_{s}^{\prime}\right)=\lambda_{M}\left(e_{1}, \ldots, e_{s}\right)$. For $k^{*}<s<m$, we have

$$
r_{M}\left(e_{1}^{\prime}, \ldots, e_{s}^{\prime}\right)=r_{M}\left(e_{1}, \ldots, e_{j}, e_{m}, e_{j+1}, \ldots, e_{s-1}\right)=r_{M}\left(e_{1}, \ldots, e_{j}, e_{j+1}, \ldots, e_{s-1}\right),
$$

as $e_{m} \in \mathrm{cl}_{M}\left(e_{1}, \ldots, e_{k^{*}}\right) \subset \mathrm{cl}_{M}\left(e_{1}, \ldots, e_{s-1}\right)$. And as in (3.3), $r_{M}\left(e_{s+1}^{\prime}, \ldots, e_{n}^{\prime}\right) \leq$ $r_{M}\left(e_{s}, \ldots, e_{n}\right)$. Hence, $\lambda\left(e_{1}^{\prime}, \ldots, e_{s}^{\prime}\right) \leq \lambda\left(e_{1}, \ldots, e_{s-1}\right)$.

We are left with $j+1 \leq s \leq k^{*}$. Note that by choice of $k^{*}, e_{m} \notin \mathrm{cl}_{M}\left(e_{1}, \ldots, e_{s-1}\right)$. Therefore,

$$
\begin{aligned}
r_{M}\left(e_{1}^{\prime}, \ldots, e_{s}^{\prime}\right) & =r_{M}\left(e_{1}, \ldots, e_{j}, e_{m}, e_{j+1}, \ldots, e_{s-1}\right) \\
& =1+r_{M}\left(e_{1}, \ldots, e_{j}, e_{j+1}, \ldots, e_{s-1}\right)
\end{aligned}
$$

Since (3.3) again applies, we have that

$$
\lambda_{M}\left(e_{1}^{\prime}, \ldots, e_{s}^{\prime}\right) \leq 1+\lambda_{M}\left(e_{1}, \ldots, e_{s-1}\right) .
$$


Observe that, since $e_{j+1} \notin \mathrm{cl}_{M}\left(e_{1}, \ldots, e_{j}\right)$, by Lemma 3.6(a),

$$
\lambda_{M}\left(e_{1}, \ldots, e_{j+1}\right)=\lambda_{M}\left(e_{1}, \ldots, e_{j}\right)+1 .
$$

Furthermore, by choice of $k^{*}, e_{m} \notin \mathrm{cl}_{M}\left(e_{1}, \ldots, e_{k^{*}-1}\right)$, but $e_{m} \in \operatorname{cl}_{M}\left(e_{1}, \ldots, e_{k^{*}}\right)$, which together imply that $e_{k^{*}} \notin \operatorname{cl}_{M}\left(e_{1}, \ldots, e_{k^{*}-1}\right)$. Hence, again by Lemma 3.6(a),

$$
\lambda_{M}\left(e_{1}, \ldots, e_{k^{*}}\right)=\lambda_{M}\left(e_{1}, \ldots, e_{k^{*}-1}\right)+1 .
$$

Therefore, from (3.4)-(3.6), we find that for $s=j+1$ or $s=k^{*}$, we have $\lambda_{M}\left(e_{1}^{\prime}, \ldots, e_{s}^{\prime}\right) \leq$ $\lambda_{M}\left(e_{1}, \ldots, e_{s}\right)$.

We claim that for $j+1<s<k^{*}$, we have $\lambda_{M}\left(e_{1}, \ldots, e_{s-1}\right) \leq \lambda_{M}\left(e_{1}, \ldots, e_{s}\right)$, so that by induction, $\lambda_{M}\left(e_{1}, \ldots, e_{s-1}\right) \leq \lambda_{M}\left(e_{1}, \ldots, e_{k^{*}-1}\right)$. This would then imply, via (3.4) and (3.6), that $\lambda_{M}\left(e_{1}^{\prime}, \ldots, e_{s}^{\prime}\right) \leq \lambda_{M}\left(e_{1}, \ldots, e_{k^{*}}\right)$. Thus, for any $s \in[n]$, we have a $t \in[n]$ such that $\lambda\left(e_{1}^{\prime}, \ldots, e_{s}^{\prime}\right) \leq \lambda\left(e_{1}, \ldots, e_{t}\right)$. Therefore, $w_{M}\left(e_{1}^{\prime}, \ldots, e_{n}^{\prime}\right) \leq$ $w_{M}\left(e_{1}, \ldots, e_{n}\right)$, which would complete the proof of the lemma.

To prove our claim, it is enough to show that when $j+1<s<k^{*}$, we have $e_{s} \in$ $\mathrm{cl}_{M}\left(e_{s+1}, \ldots, e_{n}\right)$. Indeed, it then follows from Lemma 3.6(b) that $\lambda_{M}\left(e_{1}, \ldots, e_{s}\right) \geq$ $\lambda_{M}\left(e_{1}, \ldots, e_{s-1}\right)$. So, suppose that $e_{s} \notin \mathrm{cl}_{M}\left(e_{s+1}, \ldots, e_{n}\right)$ for some $j+1<s<k^{*}$. Then, $e_{s}=r_{u v}$ for some $u, v \in \bar{V}$. (Otherwise, if $e_{s}=l_{u v}$, then since $r_{u v}>l_{u v}$, we would have $e_{s} \in \operatorname{cl}_{M}\left(e_{s+1}, \ldots, e_{n}\right)$.) Note that $l_{u v} \notin\left\{e_{1}, \ldots, e_{j}\right\}$; otherwise, the fact that $\left\{e_{1}, \ldots, e_{j}\right\}$ is a flat of $M$ would imply that $r_{u v} \in\left\{e_{1}, \ldots, e_{j}\right\}$. So, $l_{u v} \in\left\{e_{j+1}, \ldots, e_{s-1}\right\}$.

Suppose that $e_{s}=r_{x v}$ for some $v \in \bar{V}$. Then, $l_{x v} \in\left\{e_{j+1}, \ldots, e_{s-1}\right\}$, which contradicts the choice of $k^{*}$. Therefore, $e_{s} \notin R_{x}$, meaning that $e_{s}=r_{u v}$ for some $u, v \in V$.

Now, if $l_{x u}, l_{x v} \in\left\{e_{s+1}, \ldots, e_{n}\right\}$, then $e_{s} \in \mathrm{cl}_{M}\left(e_{s+1}, \ldots, e_{n}\right)$, as $\left(l_{x u}, l_{x v}, r_{u v}\right)$ is a triangle in $\overline{\mathcal{G}}$. So, WLOG, $l_{x u} \notin\left\{e_{s+1}, \ldots, e_{n}\right\}$. By choice of $k^{*}, l_{x u} \notin\left\{e_{j+1}, \ldots, e_{s}\right\}$. Therefore, $l_{x u} \in\left\{e_{1}, \ldots, e_{j}\right\}$. But now, $l_{x v} \in \mathrm{cl}_{M}\left(e_{1}, \ldots, e_{s-1}\right)$, as $\left(l_{x u}, l_{u v}, l_{x v}\right)$ is a triangle in $\overline{\mathcal{G}}$. However, $l_{x v} \notin\left\{e_{1}, \ldots, e_{j}\right\}$; otherwise, we would have $l_{x u}, l_{x v} \in$ $\left\{e_{1}, \ldots, e_{j}\right\}$, which, since $\left\{e_{1}, \ldots, e_{j}\right\}$ is a flat and $\left(l_{x u}, l_{u v}, l_{x v}\right)$ is a triangle, would imply that $l_{u v} \in\left\{e_{1}, \ldots, e_{j}\right\}$. Thus, $l_{x v}=e_{m^{*}}$ for some $m^{*}>j$. As already noted, $l_{x v} \in \mathrm{cl}_{M}\left(e_{1}, \ldots, e_{s-1}\right)$, and so once again, our choice of $k^{*}$ is contradicted.

Therefore, our assumption that $e_{s} \notin \operatorname{cl}_{M}\left(e_{s+1}, \ldots, e_{n}\right)$ always leads to a contradiction, from which we conclude that the assumption is false. This completes the proof of the lemma.

We can now furnish the last remaining piece of the proof of Proposition 3.1

Lemma 3.9. $p w(M) \geq p w(\overline{\mathcal{G}})$.

Proof. Let $\left(e_{1}, \ldots, e_{n}\right)$ be an optimal ordering of $\bar{E}$. WLOG, $\left(e_{1}, \ldots, e_{n}\right)$ may be assumed to be normal. Let $\left(e_{1}^{*}, \ldots, e_{n}^{*}\right)$ be the output of the Re-ordering Algorithm in the response to the input $\left(e_{1}, \ldots, e_{n}\right)$. Then, $\left(e_{1}^{*}, \ldots, e_{n}^{*}\right)$ has the properties listed in Claim 3.7, and, by Lemma 3.8, is also an optimal ordering of $\bar{E}$.

Now, $\left(e_{1}^{*}, \ldots, e_{n}^{*}\right)$ induces an ordered partition $\left(L_{1}, A_{1}, B_{1}, R_{1}, \ldots, L_{t}, A_{t}, B_{t}, R_{t}\right)$ of $\bar{E}$, as in Claim 3.7(a). For $j \in[t]$, define $Y_{j}=\bigcup_{i<j}\left(L_{i} \cup A_{i} \cup B_{i} \cup R_{i}\right) \cup\left(L_{j} \cup A_{j}\right)$, and $Y_{j}^{\prime}=\bar{E}-Y_{j}=\bigcup_{i>j}\left(L_{i} \cup A_{i} \cup B_{i} \cup R_{i}\right) \cup\left(B_{j} \cup R_{j}\right)$. Letting $\overline{\mathcal{G}}\left[Y_{j}\right]$ and $\overline{\mathcal{G}}\left[Y_{j}^{\prime}\right]$ denote the subgraphs of $\overline{\mathcal{G}}$ induced by $Y_{j}$ and $Y_{j}^{\prime}$, respectively, set $V_{j}=V\left(\overline{\mathcal{G}}\left[Y_{j}\right]\right) \cap V\left(\overline{\mathcal{G}}\left[Y_{j}^{\prime}\right]\right)$. In other words, $V_{j}$ is the set of vertices common to both $\overline{\mathcal{G}}\left[Y_{j}\right]$ and $\overline{\mathcal{G}}\left[Y_{j}^{\prime}\right]$. It is easily checked that $\mathcal{V}=\left(V_{1}, \ldots, V_{t}\right)$ is a path-decomposition of $\overline{\mathcal{G}}$. Note that

$$
\left|V_{j}\right|=\left|V\left(\overline{\mathcal{G}}\left[Y_{j}\right]\right)\right|+\left|V\left(\overline{\mathcal{G}}\left[Y_{j}^{\prime}\right]\right)\right|-|\bar{V}|
$$


We next observe that $\overline{\mathcal{G}}\left[Y_{j}\right]$ and $\overline{\mathcal{G}}\left[Y_{j}^{\prime}\right]$ are connected graphs. From Claim $3.7(\mathrm{~b})$, we have that $Y_{j} \subset \operatorname{cl}_{M}\left(\bigcup_{i \leq j} L_{i}\right)$. Therefore, for any edge $l_{u v}$ (or $\left.r_{u v}\right)$ in $Y_{j}-\bigcup_{i<j} L_{i}$, both $l_{x u}$ and $l_{x v}$ must be in some $L_{i}, i \leq j$. Thus, in $\overline{\mathcal{G}}\left[Y_{j}\right]$, each vertex $v \neq x$ is adjacent to $x$, which shows that $\overline{\mathcal{G}}\left[Y_{j}\right]$ is connected.

Consider any vertex $v \neq x$ in $\overline{\mathcal{G}}\left[Y_{j}^{\prime}\right]$, such that $r_{x v} \notin Y_{j}^{\prime}$. Then, $r_{u v} \in Y_{j}^{\prime}$ for some $u \neq x$. So, $r_{u v} \in B_{k}$ for some $k \geq j$. By Claim 3.7(b), $r_{u v} \in \mathrm{cl}_{M}\left(\bigcup_{i<k} L_{i}\right)-$ $\operatorname{cl}_{M}\left(\bigcup_{i<k} L_{i}\right)$. This implies that either $l_{x u} \in L_{k}$ or $l_{x v} \in L_{k}$. Hence, either $r_{x u} \in R_{k}$ or $r_{x v} \in R_{k}$. However, $r_{x v}$ cannot be in $R_{k}$, since $r_{x v} \notin Y_{j}^{\prime}$, and so, $r_{x u} \in R_{k}$. Thus, $\left(r_{x u}, r_{u v}\right)$ forms a path in $\overline{\mathcal{G}}\left[Y_{j}^{\prime}\right]$ from $x$ to $v$. It follows that $\overline{\mathcal{G}}\left[Y_{j}^{\prime}\right]$ is connected.

Therefore,

$$
\begin{aligned}
\lambda_{M}\left(Y_{j}\right) & =r_{M}\left(Y_{j}\right)+r_{M}\left(Y_{j}^{\prime}\right)-r_{M}(\bar{E}) \\
& =\left(\left|V\left(\overline{\mathcal{G}}\left[Y_{j}\right]\right)\right|-1\right)+\left(\left|V\left(\overline{\mathcal{G}}\left[Y_{j}^{\prime}\right]\right)\right|-1\right)-(\bar{V}-1)=\left|V_{j}\right|-1 .
\end{aligned}
$$

Hence,

$$
\operatorname{pw}(M)=w_{M}\left(e_{1}^{*}, \ldots, e_{n}^{*}\right) \geq \max _{j \in[t]} \lambda_{M}\left(Y_{j}\right)=\max _{j \in[t]}\left|V_{j}\right|-1=w_{\overline{\mathcal{G}}}(\mathcal{V}) \geq \operatorname{pw}(\overline{\mathcal{G}}),
$$

which proves the lemma.

The proof of Proposition 3.1 is now complete.

4. Matroids of Bounded Pathwidth. Theorem 3.2 shows that the following decision problem is NP-complete.

\section{Problem: Matroid Pathwidth}

Let $\mathbb{F}$ be a fixed field.

Instance: $\quad$ An $m \times n$ matrix $A$ over $\mathbb{F}$, and an integer $w>0$.

Question: Is there an ordering $\left(e_{1}, \ldots, e_{n}\right)$ of the elements of $M=M[A]$, such that $w_{M}\left(e_{1}, \ldots, e_{n}\right) \leq w$ ?

Similarly, Corollary 3.3 shows that the corresponding decision problem for code trelliswidth (over a fixed finite field $\mathbb{F}$ ) is NP-complete.

In this section, we consider the situation when the parameter $w$ above is a fixed constant, and therefore, not considered to be part of the problem instance. In contrast to the NP-completeness of MATROID PATHWIDTH, we believe that the following decision problem and its coding-theoretic counterpart are solvable in polynomial time.

Problem: Weak Matroid Pathwidth

Let $\mathbb{F}_{q}=G F(q)$ be a fixed finite field, and $w$ a fixed positive integer.

Instance: An $m \times n$ matrix $A$ over $\mathbb{F}_{q}$.

Question: Is there an ordering $\left(e_{1}, \ldots, e_{n}\right)$ of the elements of $M=M[A]$, such that $w_{M}\left(e_{1}, \ldots, e_{n}\right) \leq w$ ?

Our optimism above stems from the fact that the property of having pathwidth bounded by $w$ is preserved by the minors of a matroid. To be precise, let $\mathcal{P}_{w, q}$ be the class of matroids representable over the finite field $\mathbb{F}_{q}=G F(q)$, that have pathwidth at most $w$. By Lemma 2.1. $\mathcal{P}_{w, q}$ is minor-closed. Since pathwidth is an upper bound on the branchwidth of a matroid, all matroids in $\mathcal{P}_{w, q}$ have branchwidth at most $w$. Now, Geelen and Gerards have shown that if $\mathcal{M}$ is any minor-closed class of $\mathbb{F}_{q}$-representable matroids having bounded branchwidth, then $\mathcal{M}$ has finitely many excluded minors [8, Theorem 1.4]. As a result, we have the following theorem. 
TheOrem 4.1. For any integer $w>0$ and finite field $\mathbb{F}_{q}, \mathcal{P}_{w, q}$ has finitely many excluded minors. Consequently, the code family

$$
\mathfrak{C}\left(\mathcal{P}_{w, q}\right)=\left\{\mathcal{C}: \mathcal{C} \text { is a linear code over } \mathbb{F}_{q} \text { such that } t w(\mathcal{C}) \leq w\right\} .
$$

also has finitely many excluded minors.

Theorem 4.1] shows that deciding whether or not a given $\mathbb{F}_{q}$-representable matroid $M$ belongs to $\mathcal{P}_{w, q}$ can be accomplished by testing whether or not $M$ contains as a minor one of the finitely many excluded minors of $\mathcal{P}_{w, q}$. The Minor-Recognition Conjecture of Geelen, Gerards and Whittle [6, Conjecture 1.3] states that, for any fixed $\mathbb{F}_{q}$-representable matroid $N$, testing a given $\mathbb{F}_{q}$-representable matroid for the presence of an $N$-minor can be done in polynomial time. So, if this conjecture is true - and there is evidence to support its validity [ $\left[\right.$ ] then membership of an $\mathbb{F}_{q}$-representable matroid in the class $\mathcal{P}_{w, q}$ can be decided in polynomial time. Hence, assuming the validity of the Minor-Recognition Conjecture, WEAK Matroid Pathwidth is solvable in polynomial time.

While the finiteness of the list of excluded minors for $\mathcal{P}_{w, q}$ implies, modulo the Minor-Recognition Conjecture, the existence of a polynomial-time algorithm for Weak Matroid PathwidTh, an actual implementation of such an algorithm would require the explicit determination of the excluded minors. As a relatively easy exercise, we prove the following theorem.

Theorem 4.2. A matroid is in $\mathcal{P}_{1, q}$ iff it contains no minor isomorphic to any of the matroids $U_{2,4}, M\left(K_{4}\right), M\left(K_{2,3}\right)$ and $M^{*}\left(K_{2,3}\right)$.

We first verify the easy "only if" part of the above theorem.

Proposition 4.3. $U_{2,4}, M\left(K_{4}\right), M\left(K_{2,3}\right)$ and $M^{*}\left(K_{2,3}\right)$ are not in $\mathcal{P}_{1, q}$.

Proof. If $\left(e_{1}, e_{2}, e_{3}, e_{4}\right)$ is any ordering of the elements of $M=U_{2,4}$, then $\lambda_{M}\left(e_{1}, e_{2}\right)=r_{M}\left(e_{1}, e_{2}\right)+r_{M}\left(e_{3}, e_{4}\right)-\operatorname{rank}(M)=2+2-2=2$. It follows that $\operatorname{pw}\left(U_{2,4}\right)=2$.

Now consider $M=M\left(K_{4}\right)$. For any ordering $\left(e_{1}, \ldots, e_{6}\right)$ of $E\left(K_{4}\right)$, we have $r_{M}\left(e_{1}, e_{2}, e_{3}\right) \geq 2$, with equality iff $\left\{e_{1}, e_{2}, e_{3}\right\}$ is a triangle, in which case $\left\{e_{4}, e_{5}, e_{6}\right\}$ is a triad. It follows that $r_{M}\left(e_{1}, e_{2}, e_{3}\right)+r_{M}\left(e_{4}, e_{5}, e_{6}\right) \geq 5$. Hence, $w_{M}\left(e_{1}, \ldots, e_{6}\right) \geq$ $\lambda_{M}\left(e_{1}, e_{2}, e_{3}\right) \geq 2$.

The proof for $M=M\left(K_{2,3}\right)$ is very similar. For any $J \subset E\left(K_{2,3}\right)$ with $|J|=3$, $r_{M}(J)=3$, since $K_{2,3}$ has no circuits of size less than 4 . Therefore, for any ordering $\left(e_{1}, \ldots, e_{6}\right)$ of $E\left(K_{2,3}\right), w_{M}\left(e_{1}, \ldots, e_{6}\right) \geq \lambda_{M}\left(e_{1}, e_{2}, e_{3}\right)=3+3-4=2$. Thus, $\operatorname{pw}\left(M\left(K_{2,3}\right)\right) \geq 2$, and by duality, $\operatorname{pw}\left(M^{*}\left(K_{2,3}\right)\right) \geq 2$ as well.

We now prove the "if" part of Theorem 4.2, For the duration of the proof, we take $M$ to be a matroid that contains no minor isomorphic to the matroids listed in the statement of the theorem. Since $M\left(K_{4}\right)$ is a minor of each of the matroids $F_{7}, F_{7}^{*}, M\left(K_{5}\right), M^{*}\left(K_{5}\right), M\left(K_{3,3}\right)$ and $M^{*}\left(K_{3,3}\right), M$ contains none of these as minors. Therefore, $M=M(\mathcal{G})$ for some planar graph $\mathcal{G}$ (cf. [15, Theorem 13.3.1 and Proposition 5.2.6]). Evidently, we may take $\mathcal{G}$ to be connected as a graph.

Since $\mathcal{P}_{1, q}$ is closed under direct sums, we may assume that $M$ is 2 -connected. Therefore, $\mathcal{G}$ is either a graph consisting of a single vertex with a self-loop incident with it, or $\mathcal{G}$ is a loopless graph. In the former case, $M \cong U_{0,1}$, which is in $\mathcal{P}_{1, q}$. So, we may assume that $\mathcal{G}$ is loopless. If $\mathcal{G}$ has exactly two vertices, then $M \cong U_{1, n}$ for 


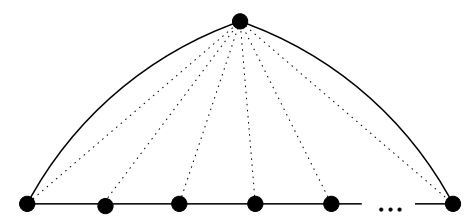

FIG. 4.1. An "umbrella" graph. A dotted line between a pair of vertices represents zero or more parallel edges between them.

some $n$, which is also in $\mathcal{P}_{1, q}$. Hence, we may assume that $|V(\mathcal{G})| \geq 3$, in which case, $\mathcal{G}$ is 2 -connected as a graph [15, Corollary 8.2.2]. Moreover, if $\mathcal{G}^{*}$ is any geometric dual of $\mathcal{G}$, then, since $M^{*}=M\left(\mathcal{G}^{*}\right)$ is 2-connected, by the same argument as above, we may assume that $\mathcal{G}^{*}$ is also 2 -connected as a graph.

At this point, we need the following definition. We call a graph an umbrella if it is of the form shown in Figure 4.1. Formally, an umbrella is a graph $H$ that consists of a circuit on $m+1$ vertices $u_{0}, u_{1}, \ldots, u_{m}$, and in addition, for each $i \in[m]$, zero or more parallel edges between $u_{0}$ and $u_{i}$. Note that $H-u_{0}$ is a simple path, where $H-u_{0}$ denotes the graph obtained from $H$ by deleting the vertex $u_{0}$ and all edges incident with it.

Returning to our proof, we have $M=M(\mathcal{G})$ for a loopless, 2-connected, planar graph $\mathcal{G}$, such that any geometric dual of $\mathcal{G}$ is also 2-connected.

LEMmA 4.4. $\mathcal{G}$ has a geometric dual $\mathcal{G}^{*}$ that is isomorphic to an umbrella.

We prove the lemma using the concept of an outerplanar graph. A planar graph is said to be outerplanar if it has a planar embedding in which every vertex lies on the exterior (unbounded) face. We will refer to such a planar embedding of the graph as an outerplanar embedding. Outerplanar graphs were characterized by Chartrand and Harary [3] as graphs that do not contain $K_{4}$ or $K_{2,3}$ as a minor.

Proof of Lemma 4.4 Since $M(\mathcal{G})$ contains no $M\left(K_{4}\right)$ - or $M\left(K_{2,3}\right)$-minor, $\mathcal{G}$ cannot contain $K_{4}$ or $K_{2,3}$ as a minor. Therefore, by the Chartrand-Harary result mentioned above, $\mathcal{G}$ is outerplanar. Let $\mathcal{G}^{*}$ be the geometric dual of an outerplanar embedding of $\mathcal{G}$.

Let $x$ be the vertex of $\mathcal{G}^{*}$ corresponding to the exterior face of the outerplanar embedding of $\mathcal{G}$. By a result of Fleischner et al. 4, Theorem 1], $\mathcal{G}^{*}-x$ is a forest. In fact, since $\mathcal{G}^{*}$ is 2 -connected, $\mathcal{G}^{*}-x$ is a tree.

We claim that no vertex of $\mathcal{G}^{*}-x$ has degree greater than two, and hence, $\mathcal{G}^{*}-x$ is a simple path. Indeed, suppose that $\mathcal{G}^{*}-x$ has a vertex $u$ adjacent to three other vertices $v_{1}, v_{2}, v_{3}$. Since $G^{*}$ is 2 -connected, there are paths $\pi_{1}, \pi_{2}$ and $\pi_{3}$ in $\mathcal{G}^{*}$ from $v_{1}, v_{2}$ and $v_{3}$, respectively, to $x$ that do not pass through $u$. Also, since $\mathcal{G}^{*}-x$ is a tree, these paths must be internally disjoint in $\mathcal{G}^{*}$. The graph $\mathcal{G}^{*}$ thus has a subgraph as depicted in Figure 4.2 But this subgraph is obviously contractible to $K_{2,3}$, and hence $\mathcal{G}^{*}$ has $K_{2,3}$ as a minor. However, this is impossible, as $M^{*}=M\left(\mathcal{G}^{*}\right)$ does not have $M\left(K_{2,3}\right)$ as a minor.

Thus, $\mathcal{G}^{*}-x$ is a simple path. The two degree-one vertices (end-points) of this path must be adjacent to $x$ in $\mathcal{G}^{*}$; otherwise, $\mathcal{G}^{*}$ is not 2 -connected. It follows that $\mathcal{G}^{*}$ is isomorphic to an umbrella.

To complete the proof of Theorem 4.2. we show that $M\left(\mathcal{G}^{*}\right) \in \mathcal{P}_{1, q}$, so that by 


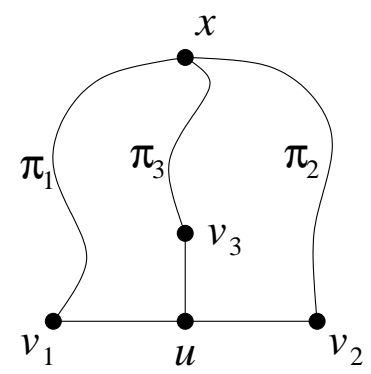

FIG. 4.2. If $\mathcal{G}^{*}-x$ has a vertex of degree at least 3 , then $\mathcal{G}^{*}$ has a $K_{2,3}$ minor.

duality, $M=M^{*}\left(\mathcal{G}^{*}\right) \in \mathcal{P}_{1, q}$. This is done by the following lemma.

Lemma 4.5. If $H$ is an umbrella, then $M(H) \in \mathcal{P}_{1, q}$.

Proof. Let $H$ be an umbrella on $m+1$ vertices $u_{0}, u_{1}, \ldots, u_{m}$, where $u_{0}$ is the vertex such that $H-\left\{u_{0}\right\}$ is a simple path. For $i \in[m]$, let $E_{i}$ denote the set of edges between $u_{0}$ and $u_{i}$. Also, for $j \in[m-1]$, let $e_{j}$ denote the edge between $u_{j}$ and $u_{j+1}$. Consider any ordering of the edges of $H$ that induces the ordered partition

$$
\left(E_{1}, e_{1}, E_{2}, e_{2}, \ldots, E_{m-1}, e_{m-1}, E_{m}\right) .
$$

Let $J=\left(\bigcup_{i=1}^{j-1}\left(E_{i} \cup\left\{e_{i}\right\}\right)\right) \cup X$, with $X \subset E_{j}$ ( $X$ may be empty). Note that the subgraph, $H[J]$, of $H$ induced by the edges in $J$ is incident only with vertices in $\left\{u_{0}, u_{1}, \ldots, u_{j}\right\}$. Therefore, setting $M=M(H), r_{M}(J)=|V(H[J])|-1 \leq j$. Similarly, the subgraph of $H$ induced by the edges in $E(H)-J$ is incident only with vertices in $\left\{u_{j}, u_{j+1}, \ldots, u_{m}, u_{0}\right\}$, and so, $r_{M}(E(H)-J) \leq m-j+1$.

Thus, $\lambda_{M}(J) \leq j+(m-j+1)-m=1$, and it follows that $\operatorname{pw}(M) \leq 1$. Being graphic, $M$ is $\mathbb{F}_{q}$-representable, and hence, $M \in \mathcal{P}_{1, q}$. $\square$

This completes the proof of Theorem 4.2 .

As a corollary to the theorem, we give a coding-theoretic characterization of the code family $\mathfrak{C}\left(\mathcal{P}_{1, q}\right)$. In coding theory, an $\mathbb{F}_{q}$-representation of a uniform matroid is called a maximum-distance separable $(M D S)$ code. For any field $\mathbb{F}_{q}$, the matrices $G_{4}, G_{2,3}$ and $G_{2,3}^{*}$ below are $\mathbb{F}_{q}$-representations of $M\left(K_{4}\right), M\left(K_{2,3}\right)$ and $M^{*}\left(K_{2,3}\right)$, respectively.

$$
\begin{gathered}
G_{4}=\left[\begin{array}{cccccc}
1 & 0 & 0 & 1 & 0 & -1 \\
0 & 1 & 0 & 1 & 1 & -1 \\
0 & 0 & 1 & 0 & 1 & -1
\end{array}\right] ; \\
G_{2,3}=\left[\begin{array}{cccccc}
1 & 0 & 0 & 0 & -1 & -1 \\
0 & 1 & 0 & 0 & 1 & 0 \\
0 & 0 & 1 & 0 & 0 & 1 \\
0 & 0 & 0 & 1 & 1 & 1
\end{array}\right] ; \quad G_{2,3}^{*}=\left[\begin{array}{cccccc}
1 & -1 & 0 & -1 & 1 & 0 \\
1 & 0 & -1 & -1 & 0 & 1
\end{array}\right] .
\end{gathered}
$$

The matroids $M\left(K_{4}\right), M\left(K_{2,3}\right)$ and $M^{*}\left(K_{2,3}\right)$, being binary, are uniquely representable over $\mathbb{F}_{q}$, in the matroid-theoretic sense [15, Section 6.3 and Theorem 10.1.3]. We let $\mathcal{C}\left(K_{4}\right), \mathcal{C}\left(K_{2,3}\right)$ and $\mathcal{C}\left(K_{2,3}\right)^{\perp}$ denote the codes over $\mathbb{F}_{q}$ generated by the matrices $G_{4}, G_{2,3}$ and $G_{2,3}^{*}$, respectively. 

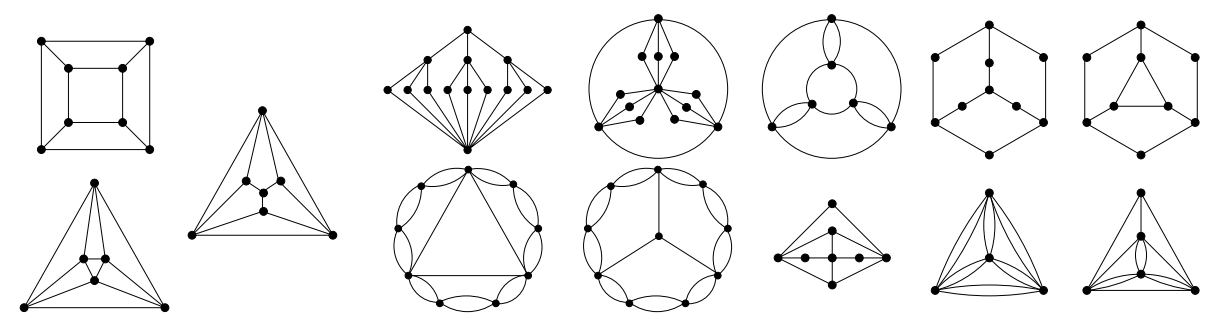

FIG. 4.3. Some of the planar graphs whose cycle matroids are excluded minors for $P_{2, q}$.

Corollary 4.6. Let $\mathbb{F}_{q}$ be an arbitrary finite field. A linear code $\mathcal{C}$ over $\mathbb{F}_{q}$ has trellis-width at most one iff it contains no minor equivalent to any of the following:

(i) a $[4,2] M D S$ code;

(ii) a code obtainable by applying an automorphism of $\mathbb{F}_{q}$ to one of the codes $\mathcal{C}\left(K_{4}\right), \mathcal{C}\left(K_{2,3}\right)$ and $\mathcal{C}\left(K_{2,3}\right)^{\perp}$.

The problem of finding the complete set of excluded minors for $\mathcal{P}_{w, q}$ quickly becomes difficult for $w>1$. The main obstacle is that we may only assume the basic property of 2-connectedness for such excluded minors. The class $\mathcal{P}_{w, q}$ is not even closed under 2-sums, so excluded minors for the class need not be 3 -connected. An illustration of this is given by the following result, which provides a partial list of excluded minors for $\mathcal{P}_{2, q}$.

Proposition 4.7. For any finite field $\mathbb{F}_{q}$, the matroids $F_{7}, F_{7}^{*}, M\left(K_{5}\right), M^{*}\left(K_{5}\right)$, $M\left(K_{3,3}\right), M^{*}\left(K_{3,3}\right)$, and $M(\mathcal{G})$, where $\mathcal{G}$ is any of the planar graphs in Figure 4.3. are excluded minors for $\mathcal{P}_{2, q}$. If $q \geq 4$, then $U_{3,6}$ is also an excluded minor for $\mathcal{P}_{2, q}$.

We omit the proof, as it is only a matter of verifying that for each matroid $M$ listed in the proposition, $M \notin \mathcal{P}_{2, q}$, but $M \backslash e, M / e \in \mathcal{P}_{2, q}$ for any $e \in E(M)$. We point out that the cycle matroids of all but the three leftmost graphs in Figure 4.3 are not 3 -connected.

Acknowledgment. The author would like to thank Jim Geelen for contributing some of his ideas to this paper, and Alexander Vardy for pointers to the prior literature on trellis complexity.

\section{REFERENCES}

[1] S. Arnborg, D.G. Corneil and A. Proskurowski, "Complexity of finding embeddings in a $k$-tree," SIAM J. Alg. Disc. Meth., vol. 8, pp. 277-284, 1987.

[2] H.L. Bodlaender, "A tourist guide through treewidth," Acta Cybernetica, vol. 11, pp. 1-23, 1993.

[3] G. Chartrand and F. Harary, "Planar permutation graphs," Ann. Inst. Henri Poincaré Sec. B, vol. III, no. 4, pp. 433-438, 1967.

[4] H.J. Fleischner, D.P. Geller and F. Harary, "Outerplanar graphs and weak duals," J. Indian Math. Soc., vo. 38, pp. 215-219, 1974.

[5] G.D. Forney Jr., "Dimension/length profiles and trellis complexity of linear block codes," IEEE Trans. Inform. Theory, vol. 40, no. 6, pp. 1741-1752, Nov. 1994.

[6] J. Geelen, B. Gerards and G. Whittle, "Towards a matroid-minor structure theory", to appear in Combinatorics, Complexity and Chance. A tribute to Dominic Welsh, G. Grimmett and C. McDiarmid, eds., Oxford University Press, 2007. Available online at http://homepages.cwi.nl/ bgerards/personal/papers/towards_welsh.pdf. 
[7] J. Geelen, B. Gerards and G. Whittle, "On Rota's Conjecture and excluded minors containing large projective geometries," J. Combin. Theory, Ser. B, vol. 96, pp. 405-425, 2006.

[8] J. Geelen and G. Whittle, "Branch-width and Rota's Conjecture," J. Combin. Theory, Ser. B, vol. 86, no. 2, pp. 315-330, Nov. 2002.

[9] R. Hall, J. Oxley and C. Semple, "The structure of 3-connected matroids of path width three", Europ. J. Combin., vol. 28, pp. 964-989, 2007.

[10] G.B. Horn and F.R. Kschischang, "On the intractability of permuting a block code to minimize trellis complexity," IEEE Trans. Inform. Theory, vol. 42, no. 6, pp. 2042-2048, Nov. 1996.

[11] K. Jain, I. Măndoiu and V.V. Vazirani, "The 'art of trellis decoding' is computationally hard — for large fields," IEEE. Trans. Inform. Theory, vol. 44, no. 3, pp. 1211-1214, May 1998.

[12] N. Kashyap, "A decomposition theory for binary linear codes," submitted to IEEE Trans. Inform. Theory. ArXiv e-print cs.DM/0611028

[13] F.J. MacWilliams and N.J.A. Sloane, The Theory of Error-Correcting Codes, North-Holland, Amsterdam, 1977.

[14] D.J. Muder, "Minimal trellises for block codes," IEEE. Trans. Inform. Theory, vol. 34, no. 5, pp. 1049-1053, Sept. 1988.

[15] J.G. Oxley, Matroid Theory, Oxford University Press, Oxford, UK, 1992.

[16] N. Robertson and P.D. Seymour, "Graph minors. I. Excluding a forest," J. Combin. Theory, Ser. B, vol. 35, pp. 39-61, 1983.

[17] A. Vardy, "Trellis Structure of Codes," in Handbook of Coding Theory, R. Brualdi, C. Huffman and V. Pless, Eds., Amsterdam, The Netherlands: Elsevier, 1998. 